\title{
INTERPRETACIÓN CONSTITUCIONAL, CONTROL JUDICIAL DE LA LEY Y DESACUERDOS
}

\section{CONSTITUTIONAL INTERPRETATION, JUDICIAL REVIEW OF LEGISLATION AND DISAGREEMENTS}

\author{
ESTEBAN Buriticá-Arango*
}

RESUMEN: Algunos filósofos han sostenido tradicionalmente que la legitimidad del control judicial de la ley depende al menos en parte de la existencia de respuestas correctas en la interpretación de los derechos constitucionales, y que las posiciones escépticas sobre la indeterminación de la constitución implican también una serie de objeciones al control judicial de la ley. En este trabajo sostendré que esta tesis es equivocada y que en realidad las teorías semánticas o metaéticas acerca del significado de las cláusulas constitucionales tienen muy poco que aportar, en contextos de desacuerdos razonables, a las teorías sobre la legitimidad de cualquier procedimiento de control de constitucionalidad.

Palabras clave: control de constitucionalidad, interpretación constitucional, respuestas correctas, desacuerdos, democracia.

ABSTRACT: Some philosophers have traditionally held that the legitimacy of judicial review of legislation depends partially on the existence of right answers in the interpretation of constitutional rights, and that the skeptical positions on the indeterminacy of the constitution also imply several objections to the judicial review of legislation. In this paper it is explained that this thesis is wrong and that actually the semantic or metathetic theories of the meaning of constitutional clauses scarcely contribute, in contexts of reasonable disagreements, to the theories about the legitimacy of any procedure of judicial review.

Key words: judicial review of legislation, constitutional interpretation, right answers, disagreements, democracy.

\section{INTRODUCCIÓN}

Durante los últimos años se ha vuelto un lugar común sostener que la interpretación constitucional es una actividad esencialmente valorativa ${ }^{1}$. Suele decirse que el carácter típicamente vago y abstracto de las disposiciones constitucionales, sobre todo de aquellas que consagran derechos básicos, hace que entre el significado previo del texto constitucional y el sentido de los fallos judiciales exista siempre una "brecha interpretativa" 2 que el juez solo

\footnotetext{
Profesor de Teoría del Derecho de la Universidad de San Buenaventura, sede Medellín, Colombia. Máster en Derecho Constitucional del Centro de Estudios Políticos y Constitucionales de España. El autor desea agradecer a los árbitros anónimos designados por la revista por sus valiosos comentarios.

1 Al respecto, Ferreres Comella (2012) p. 27; Guastini (2014) p. 209; Moreso (2004) pp. 54 y ss.; Bayón (2001) p. 69; WaLdron (2005) pp. 195 y ss.; DWORKIn (1996) p. 2.

2 La expresión es de Gargarella (2012) p. 79.
} 
puede salvar acudiendo a razonamientos morales ${ }^{3}$. Para determinar, por ejemplo, si "la igualdad de trato" protege el derecho a percibir los mismos salarios, o si "el libre desarrollo de la personalidad" concede el derecho a consumir cierto tipo de drogas, no basta -según se diceuna simple subsunción del caso particular en la norma genérica, sino ante todo un razonamiento moral acerca de cuál debe ser el contenido y el alcance de esos derechos. Sobre esta idea básica se han formulado, sin embargo, profundas cuestiones teóricas en las que el amplio consenso parece diluirse. Algunas veces se afirma, desde posturas cognoscitivistas, que los juicios morales involucrados en la interpretación constitucional pueden tener un carácter objetivo, que el contenido de las disposiciones es previo a la interpretación de los jueces, y que siempre o casi siempre es posible hallar respuestas correctas en casos particulares. Otras veces, sin embargo, se sostiene desde posturas escépticas que el razonamiento moral tiene un carácter esencialmente subjetivo, que el contenido de las disposiciones constitucionales es siempre una construcción del intérprete, y que por tanto nunca o casi nunca es posible hallar esas respuestas correctas ${ }^{4}$. Esta discusión, que no desarrollaré a fondo en este escrito, ha dado lugar a un amplio debate cuyo problema central a veces pasa desapercibido.

Como han puesto de manifiesto Bayón y Waldron5, la existencia de respuestas correctas en la interpretación constitucional ha sido considerada a veces como una razón a favor o en contra de ciertos modelos de control judicial de las leyes. En términos generales, la idea es que el carácter objetivo o subjetivo de los juicios de valor involucrados en la interpretación incide necesariamente en el tipo y alcance de las competencias que debe atribuirse a los jueces para que ejerzan control de las leyes. Mientras mayor sea la certeza acerca del contenido de las disposiciones constitucionales y, por tanto, menos discrecional sea la decisión, un menor número de objeciones recaerían sobre un diseño institucional que atribuya a los jueces la última palabra sobre el contenido y el alcance de los derechos. "Si se entendiese" como señala Prieto, "que estos [los jueces] aplican fiel y rectamente la Constitución, nada habría que criticar" ${ }^{\text {. }}$ Pero, en sentido inverso, mientras mayor sea la indeterminación de esas disposiciones y menores las posibilidades de controlar la discrecionalidad del juez, habría mayores razones para delegar en otros órganos, sobre todo en un parlamento democráticamente constituido, la interpretación última de la constitución. De esta manera, las discusiones acerca de la legitimidad del control judicial de la ley parecen reducirse a veces, al menos en parte, a discusiones acerca de la naturaleza de la interpretación constitucional y de los juicios morales involucrados en ella.

En este trabajo trataré de mostrar que ambos problemas son en realidad independientes y que la certeza de que existen respuestas correctas tiene muy poco que aportar a las discusiones acerca de la legitimidad del control judicial de la ley. En la primera parte, valiéndome de un análisis detallado del argumento propuesto por Hamilton en el Federalista

\footnotetext{
3 BAYÓN (2001) p. 69: "dado que los preceptos constitucionales que declaran derechos básicos están ordinariamente formulados en términos considerablemente vagos y abstractos, su aplicación hace estrictamente inevitable una «lectura moral» de los mismos". BRINK (2015) p. 1: "Because these individual rights are typically moral and political rights, the judiciary's interpretive task requires them to make substantive moral and political commitments".

${ }^{4} \mathrm{Al}$ respecto, Guastini (2014) p. 346 y (2011), Moreso (1997) p. 183; Gargarella (1997) p. 59.

5 Ver Bayón (2001) p. 69, Waldron (2005) p. 202 y Waldron (1998) p. 75.

${ }^{6}$ Prieto Sanchís (2009) p. 162
} 
78, sostendré que la idea de que la existencia de respuestas correctas en la interpretación constitucional respalda modelos fuertes de control judicial supone a menudo una concepción errada acerca de la legitimidad de las constituciones. En la segunda parte, trataré de mostrar cómo la existencia o inexistencia de respuestas correctas en la interpretación de disposiciones constitucionales han sido usadas tradicionalmente como razones a favor o en contra del control judicial de constitucionalidad, analizando las teorías cognoscitivistas de la interpretación formuladas por Brink, Alexy y Dworkin, así como las posturas escépticas de autores como Tushnet. Finalmente, sostendré que las teorías analíticas o descriptivas de la interpretación constitucional (cuyo interés fundamental es explicar el significado de las disposiciones constitucionales) no proporcionan soluciones a una serie de problemas que la teoría de la legitimidad política debería resolver. Sobre este último punto recalcaré que la preocupación central de una teoría de la legitimidad del control de constitucionalidad no puede ser la (in)existencia de respuestas correctas en materia de derechos, sino ante todo el hecho constatable de que existen desacuerdos profundos acerca de cuáles son esas respuestas correctas y las condiciones morales que debe poseer el procedimiento diseñado para tomar una decisión justa.

\section{EL ARGUMENTO HAMILTON - MARSHALL}

El argumento según el cual la legitimidad del control judicial de la ley reside en el papel institucional que desempeñan los jueces como aplicadores ${ }^{7}$ de la constitución aparece ya en los orígenes remotos del constitucionalismo. Hamilton resumió en El Federalista 78 las razones que años después servirían al juez Marshall para justificar la práctica en Marbury v. Madison: (1) que siendo la función de los jueces la aplicación del derecho y la constitución el derecho supremo del Estado, los jueces estaban facultados para aplicar la constitución aún en aquellos casos en los que fuese incompatible con la ley, y (2) que actuando de esta manera los jueces no estarían imponiendo al legislador su propio criterio, sino más bien los límites que el pueblo había dejado establecidos en la constitución. En este sentido, el argumento supone que los jueces aplican fielmente la constitución y, por tanto, no usurpan las competencias del legislador democrático ni atentan contra el principio de separación de poderes. En palabras de Hamilton: "[My argument] by any means suppose a superiority of the judicial to the legislative power. It only supposes that the power of the people is superior to both; and that where the will of the legislature declared in its statutes, stands in opposition to that of the people declared in the constitution, the judges ought to be governed by the latter, rather than

\footnotetext{
7 Aquí debe entenderse "aplicación" en un sentido amplio, que toma en cuenta no solo la idea usual (y probablemente equivocada) de que el juez utiliza un mero silogismo para deducir la solución correcta para el caso concreto a partir de una norma, sino también aquellas teorías otras que, como veremos más adelante, consideran que el juez debe realizar un razonamiento complejo que involucra juicios morales, deducciones lógicas, tests de proporcionalidad o razonabilidad, entre otros. La tesis común a estas ideas, que me interesa destacar en este escrito, es que existen respuestas correctas en la interpretación constitucional. Aunque Hamilton no desarrolló una teoría de la interpretación constitucional, sí supone que los jueces pueden conocer "[the will/intention] of the people declared in the constitution", es decir, supone una teoría cognoscitivista. Hamilton (2005) p. 415.
} 
the former. They ought to regulate their decisions by the fundamental laws, rather than by those which are not fundamental"s.

Así planteado, el argumento de Hamilton descansa sobre dos premisas. En primer lugar, supone que la constitución posee respecto de la legislación ordinaria mayores credenciales democráticas -o, en general, mayor legitimidad política- y debe por eso ser respetada por todas las autoridades del Estado, incluyendo las mayorías legislativas. Solo de esa manera puede resguardarse la rigidez constitucional de la objeción contramayoritaria, y reclamar para los muertos -como suele decirse- autoridad sobre los vivos. En segundo lugar, el argumento supone que los jueces pueden hallar en el texto constitucional el contenido de las normas supremas (the willintention of the people) ${ }^{9}$, sin crearlo ellos mismos, de manera que no existiría ningún acto político de creación o ninguna "brecha interpretativa" importante entre el texto constitucional y las decisiones judiciales que invalidan o inaplican leyes ${ }^{10}$. Como puede advertirse, la primera premisa del argumento pretende fundamentar el carácter rígido de las constituciones, mientras que la segunda quiere mostrarnos el control judicial de la ley como un acto de aplicación y no de creación del derecho, por lo que supone una teoría cognoscitivista de la interpretación constitucional.

En las últimas décadas se han hecho una serie de críticas que muestran las debilidades de este argumento, poniendo en serias dificultades al control judicial de la ley. Esas críticas van dirigidas contra la primera premisa (i.e. la supuesta dignidad democrática de las constituciones actuales) o contra la segunda (i.e. la aplicación fiel de la constitución por parte de los jueces), tal como paso a ilustrarlo.

\section{PRIMERA PREMISA: LA LEGITIMIDAD DE LA CONSTITUCIÓN}

El éxito del argumento de Hamilton depende, en primer lugar, de que la constitución realmente refleje la "voluntad del pueblo" o, al menos, sea producto de un gran consenso popular que por sus méritos democráticos tenga prevalencia sobre las decisiones adoptadas por las mayorías legislativas. En este sentido, los límites impuestos al legislador democrático serían legítimos porque han sido creados directamente por el pueblo, una instancia democrática superior. Pero es justo aquí donde aparece el primer problema: sabemos bien que muchos procesos constituyentes, incluso algunos de gran relevancia histórica, no fueron precisamente la materialización de la igualdad y la autonomía política de los ciudadanos. En ellos no participaron directa ni indirectamente amplios grupos de la población, como los negros, las mujeres, los pobres, los esclavos, los miembros de algunos grupos étnicos, religiosos y políticos, entre otros. Muchas otras constituciones fueron impuestas por (o a instancias de) potencias coloniales, poderes de ocupación, dictadores o facciones vencedo-

\footnotetext{
8 Hamilton (2005) p. 415.

9 Hamilton (2005) p. 415: "A constitution is in fact, and must be, regarded by the judges as a fundamental law. It therefore belongs to them to ascertain its meaning as well as the meaning of any particular act proceeding from the legislative body. If there should happen to be an irreconcileable variance between the two, that which has the superior obligation and validity ought of course to be preferred; or in other words, the constitution ought to be preferred to the statute, the intention of the people to the intention of their agents".

10 Ver BAYÓN (2001) p. 69
} 
ras en conflictos internos que impidieron la participación igualitaria y autónoma de todos los sectores de la sociedad. Es por eso que, en países como Estados Unidos, cuyo proceso constituyente excluyó gran parte del "pueblo", es difícil atribuir mayores credenciales democráticas a la constitución que a la actual legislación ordinaria ${ }^{11}$.

Por supuesto, es probable que muchas constituciones, especialmente las adoptadas en años recientes ( $y$, más aun, las constituciones venideras) sean producto de un procedimiento democrático incluyente, y cobre entonces validez el argumento de Hamilton. Sin embargo, aún en estos casos es necesario hacer frente a una crítica adicional, formulada hace ya más de dos siglos por Jefferson: ¿es realmente democrático que en una sociedad prevalezca la voluntad de los muertos, plasmada en la constitución, sobre la voluntad de los vivos? El argumento de Hamilton parece suponer que mediante los procesos constituyentes son realmente las sociedades las que, en momentos lucidez, deciden autolimitarse para proteger sus derechos frente a los peligros que pueden representar sus propias decisiones futuras, tomadas quizás en momentos de debilidad. Esta es la idea que subyace a la metáfora de Ulises, formulada hace ya varias décadas por Elster ${ }^{12}$, para quien el "precompromiso" de las comunidades políticas también puede ser una estrategia racional. Así como decidió Ulises atarse al mástil de su embarcación para no ceder al canto de las sirenas, también podrían las comunidades políticas autorestringirse mediante constituciones rígidas para reguardar los derechos y principios básicos de las eventuales debilidades de las mayorías. Pero hoy sabemos que esta idea brinda una imagen errónea del problema: la sociedad que redacta la constitución, representada por las mayorías, es diferente de aquella que años o siglos después debe obedecerla. Como señala Bayón, "la sociedad no es la misma a lo largo del tiempo, con lo que hablar de "autolimitación" no pasa de ser un abuso del lenguaje"13. Es por esto que la primera premisa del argumento de Hamilton pierde fuerza: si los límites a la voluntad mayoritaria de una sociedad (presente) son impuestos por la voluntad mayoría de otra sociedad (pasada), entonces se desvanecen la igualdad y la autonomía política que hacen legítimas a las decisiones democráticas.

Aún existe, sin embargo, otra estrategia para defender el argumento de Hamilton que ha ganado bastante popularidad en la teoría política en las últimas décadas. En vez de concebir la legitimidad de la constitución en términos puramente procedimentales y exigir, por tanto, la participación igualitaria de todos los ciudadanos en la decisión constituyente, podríamos atribuirle una legitimidad sustancial que ponga el acento en el contenido. Desde este punto de vista, diríamos que la constitución no es legítima por haber sido creada mediante procedimientos democráticos, sino ante todo por incorporar un conjunto de derechos sustancialmente correctos. Podríamos decir, como lo hace Dworkin, que los derechos son un presupuesto de la democracia, que el procedimiento mayoritario es valioso precisa-

11 Ver Gargarella (1997) p. 57

12 Este es el argumento con el que ELSTER (1988) trató originalmente de fundamentar la rigidez constitucional.

13 BAyón (2001) p. 78. Ver también: Linares (2008) p. 50, Marmor (2015) p. 46, y BAyón (2002) p. 114. MARMOR (2015) p. 46: "lo que ocurre con el caso constitucional no es Ulises atándose a sí mismo al mástil, sino un Ulises que ata a otros, a sus sucesores políticos, al mástil con él. Además, a diferencia de Ulises que conoce el canto de las sirenas es una tentación mortal, no podemos saber esto en el caso constitucional y ciertamente podemos no estar de acuerdo con ello". 
mente cuando se sujeta a límites sustanciales, y que las constituciones, tal como han sido -o pueden ser- redactadas en los Estados constitucionales de hoy, reflejan adecuadamente esos límites ${ }^{14}$. Por tanto, si la democracia es un ideal valioso, también tienen que serlo por definición los derechos constitucionales: "la democracia sería en sí misma el fundamento de la limitación del poder de la mayoría" 15 . También podríamos decir, como lo hace Ferrajoli, que las constituciones son intrínsecamente valiosas solo porque materializan el ideal de los derechos y, por tanto, de la justicia. Las constituciones garantizan, según Ferrajoli, las condiciones que permiten a los seres humanos desarrollarse como sujetos morales, atrincherándolas frente a cualquier forma de poder político -incluido el de las mayorías-, y atribuyéndoles por tanto un necesario carácter incondicional e intransigible ${ }^{16}$. Los derechos deben hacer parte entonces, según fórmulas ampliamente conocidas, de una "esfera de lo indecidible" o de un "coto vedado" que solo puede configurarse exitosamente a través de una constitución cuyo contenido no esté sujeto a decisión política alguna (ni siquiera por parte de las mayorías).

Pero incluso estos argumentos no logran salvar el razonamiento de Hamilton. El primero pretende mostrarnos que la tensión entre constitución y democracia puede solucionarse con una mera estipulación conceptual que robustece la definición de "democracia”, esto es, que incorpora en su significado la idea de derechos y de constitución. En realidad, lo único que se logra demostrar con esto es la tesis trivial de que la tensión entre los derechos y el ideal del autogobierno no se da como una tensión entre la democracia y algo externo a ella, sino más bien como "una tensión enteramente interna al propio ideal democrático" ${ }^{17}$. En otras palabras, el argumento pretende hacernos ver que la constitución es legítima porque incorpora las precondiciones de la democracia, pero sin proporcionarnos una teoría acabada sobre esas precondiciones que disuelva la tensión entre el ideal de los derechos y el de la democracia. El segundo argumento hace algo similar: supone equivocadamente que entre los derechos de autonomía política y los derechos de libertad, incluidos todos en la "esfera de lo indecidible", no existen tensiones importantes que pongan en cuestión la legitimidad de la constitución, o termina admitiendo sin más -como lo hace el mismo Ferrajoli- que los derechos de autonomía política son "derechos-medio", "secundarios" o "instrumentales", que deben ser limitados para evitar transgresiones a los derechos de libertad, que serían a su vez "derechos-fines", "primarios" o "sustanciales"18.

\footnotetext{
14 Dworkin resume en estos términos su concepción de la democracia constitucional: "It denies that it is a defining goal of democracy that collective decisions always or normally be those that a majority or plurality of citizens would favor if fully informed and rational... Democracy means government subject to conditions -we call these the "democratic" conditions - of equal status for all citizens". Estas son las condiciones "that must be met before majoritarian decision making can claim any automatic moral advantage over other procedures for collective decision". Ver DWORKIN (1996) p. 17 y 23.

15 BAYÓN (2002) p. 75

16 Ver Ferrajoli (2013) p. 22.

17 BAYÓN (2002) p. 84.

18 Para Ferrajoli, los derechos primarios "designan intereses o necesidades... cuya satisfacción es un fin en sí misma", mientras que los derechos secundarios "consisten en derechos-poder y designan... el medio de la autodeterminación al que en un sistema democrático se confía la consecución de los fines jurídicos. La función de los derechos primarios sería "disciplinar el ejercicio de los poderes de autodeterminación, en otro caso ab-
} 
Pero el defecto más notable de estos argumentos es que suponen una teoría acabada de los derechos constitucionales, por lo que debemos suponer que el contenido nuestras constituciones reflejan o pueden reflejar correctamente el ideal de los derechos (de la justicia). Sin embargo, lo cierto es que -como lo ha puesto de manifiesto Waldron ${ }^{19}$ - en sociedades pluralistas e ideológicamente fragmentadas como las nuestras existen profundos desacuerdos acerca de cuáles deben ser nuestros derechos y cuál debe ser su alcance y contenido. Nuestras constituciones solo reflejan las concepciones de justicia que los miembros de un grupo compartieron en el pasado, pero difícilmente reflejan el consenso unánime de todos los ciudadanos de hoy. Y es que, desde el punto de vista de su contenido, algunos derechos pueden ser vistos por ciertos ciudadanos como imposiciones injustificadas, y no parece existir un conjunto de principios autoevidentes que nos permita saber con claridad si están equivocados o no. Entonces no solo importa cuál es el contenido de la constitución, sino también quién la creó y cómo fue creada ${ }^{20}$ : importa saber, en otras palabras, cómo fueron excluidas ciertas concepciones de los derechos para privilegiar otras que, una vez consignadas en la constitución, ya no pueden ser modificadas a través de procedimientos mayoritarios. Para evaluar, en últimas, la legitimidad de nuestras constituciones, no basta con postular una teoría los derechos, sino además una que permita justificar la legitimidad del procedimiento a través del cual fue creada. Sobre este punto volveré más adelante.

\section{SEGUNDA PREMISA: RESPUESTAS CORRECTAS Y CONTROL JUDICIAL}

En décadas recientes han sido autores como Brink, Alexy y Dworkin quienes han desarrollado con mayor profundidad el tipo de cognoscitivismo que supone el argumento de Hamilton. Aunque parten de asunciones teóricas y metodológicas distintas, los tres admiten pacíficamente que el contenido de los principios constitucionales -sobre todo de aquellos que incorporan derechos- proporciona soluciones correctas para (casi) todos los casos, y que el control judicial de las leyes es, por esa razón, entre otras, un procedimiento legítimo que no lesiona los principios democráticos. Asumen, por tanto, que el contenido y el alcance de los derechos pueden ser objetivamente establecidos, y tratan de explicar las características que debe satisfacer una aplicación correcta de los mismos. Veamos con más detalle la estructura de sus argumentos.

\section{BRINK Y LA TEORÍA CAUSAL DE LA REFERENCIA}

David Brink ${ }^{21}$ señala que una explicación adecuada del significado de las disposiciones constitucionales debe poder dar cuenta de su contenido semántico (semantics) y del propósito dominante del legislador (dominant purpose). Considera, en primer lugar, que el contenido semántico de las disposiciones normativas en general, y constitucionales

solutos, en los que consisten los derechos secundarios”. Ferrajoli (2013) p. 19. Ver también: Ferrajoli (2006) p. 232 y FALLON (2008) p. 1713.

19 Ver Waldron $(1998,2005)$.

20 Al respecto, ver Dahl (1989) p. 191, Beitz (1989) pp. 77 y 95, Martí (2006) p. 138, Bayón (2002) p. 76, WALDRON (2005) y LiNARES (2008) p. 31.

21 BRINK (1988). 
en particular, no depende del uso que damos a las palabras en las cuales se formulan, sino -conforme a la teoría causal de la referencia ${ }^{22}$ - de los objetos a los que hacen referencia. Así como el significado de la palabra "agua" no depende, según Brink ${ }^{23}$, de nuestras creencias acerca de las características del líquido, sino de sus propiedades químicas y físicas, así también el significado de los términos en que se formulan las normas constitucionales dependen de los objetos a los cuales hacen referencia. De ese modo, por ejemplo, el significado de expresiones con contenido moral como "trato inhumano" o "pena degradante", previstas en varias constituciones contemporáneas, no está determinado por nuestras creencias ${ }^{24}$ acerca de su significado, sino ante todo por el conjunto de hechos objetivos que harían que una pena o un trato posean dichas características (y aquí asume Brink, claramente, un realismo ético). En palabras de Brink, "The meaning and reference of our terms is given by the way the world is -in the case of the moral and political terms found in many constitutional provisions, by certain kinds of social and political factors" 25 .

En segundo lugar, el contenido semántico de las disposiciones normativas no es en ciertos casos, según Brink, suficiente para realizar una interpretación adecuada de la constitución. Es necesario acudir entonces a los "propósitos dominantes" del constituyente, entendidos como "abstract intentions, that is, the kinds of values, policies, and principles which the framers of the law were trying to realize"26. Para Brink, como para Dworkin, el derecho está compuesto no solo por normas o reglas de primer orden que han sido creadas mediante procedimientos reconocidos en la constitución o en la regla de reconocimiento del sistema (fuentes del derecho), sino también por un conjunto de principios de carácter moral y político que subyacen, y proporcionan justificación, a esas normas de primer orden ${ }^{27}$. Una interpretación adecuada de las disposiciones constitucionales debe tomar en cuenta, en consecuencia, no solo el contenido semántico de las normas de primer orden, sino también los principios morales y políticos que subyacen a ellas.

Ahora bien, Brink echa mano de esta forma de cognoscitivismo para justificar el control judicial de la ley y hacer frente a la objeción democrática. Comienza por distinguir dos tipos de control judicial de las leyes: el interpretativo y el no interpretativo. En el primer caso, los jueces declaran la inconstitucionalidad de las leyes con base en una interpretación correcta de la constitución; en el segundo, lo hacen porque consideran que la ley es políticamente inconveniente o imprudente (unwise). El control de constitucionalidad sería legítimo, por tanto, solo en el primer caso ${ }^{28}$. Por el contrario, cuando los jueces efectúan un control no interpretativo, transgreden los principios de la democracia y la separación de poderes, de modo que las objeciones a la supremacía judicial cobrarían relevancia. De esta

\footnotetext{
22 Para una exposición detallada de estas teorías, ver Ramírez Ludeña (2015).

23 BRINK (1988) p. 177.

24 BRINK (1988) p. 118: “...the meaning or reference of our terms is not determined by anyone's beliefs".

25 BRINK (1988) p. 123.

26 BRINK (1988) p. 128.

27 BRINK (1988) p. 130: "First-order legal standards do not exist in a vacuum; they are introduced for certain reasons, perform certain social and political functions, and realice certain principles of political morality... they too are legal standards".

28 BRINK (1988) p. 136.
} 
manera, es claro que para Brink la legitimidad del control judicial de las leyes depende de que sean posibles las interpretaciones correctas de la constitución.

Por supuesto, Brink admite que "there is no guarantee that judges will not make legal mistakes or legal misinterpretations" 29 . Los jueces pueden equivocarse cuando interpretan normas constitucionales (porque no son infalibles) o, incluso, descartar conscientemente la interpretación correcta (porque no siempre son honestos). Sin embargo, insiste en que su modelo permite mostrar que muchos de los fallos de la Corte Suprema de Estados Unidos, tradicionalmente criticados por su contenido político, en realidad sí están sustentadas en una interpretación correcta de la constitución. En particular, trata de mostrar cómo Brown v. Board of Education está sustentado en una interpretación correcta del término "equal protection" ${ }^{30}$ previsto en la decimocuarta enmienda, y cómo Griswold v. Connecticut puede fundamentarse igualmente en una interpretación correcta de la primera, tercera, cuarta y novena enmienda, las cuales consagrarían -aunque no expresamente- el derecho a la privacidad.

\section{AlEXY y EL PRINCIPIO DE PROPORCIONALIDAD}

Aunque Alexy no desarrolla una teoría general de la interpretación constitucional (i.e. una teoría del significado de las disposiciones constitucionales), considera que es posible determinar el alcance de los principios, concebidos como "mandatos de optimización", aplicando los principios de idoneidad, necesidad y proporcionalidad en sentido estricto. El principio de idoneidad exige que la decisión adoptada por el juez esté orientada específicamente a la protección del principio que posee mayor peso; el principio de necesidad, por su parte, exige que la vulneración de uno de los principios en pugna sea necesario para la protección del otro, es decir, que no existan alternativas menos lesivas; y el principio de proporcionalidad en sentido estricto exige, finalmente, que "cuanto más alto sea el grado de incumplimiento o menoscabo de un principio, tanto mayor debe ser la importancia del cumplimiento del otro"31, por lo que es necesario efectuar una ponderación para comparar el peso de cada principio en el caso concreto y determinar cuál de los dos debe prevalecer. El principio de proporcionalidad hace parte de una teoría de la argumentación jurídica más general que, en palabras de Alexy, "formula reglas o condiciones de argumentación o decisión práctica racional" 32 .

Entendido en estos términos, el principio de proporcionalidad posibilitaría la aplicación racional de derechos. Es posible, en opinión del autor, atribuir peso a los derechos o determinar su grado de afectación de manera objetiva, dado que, como señala el mismo Alexy, "rational argument and, thereby, objectivity is possible in constitutional argumentation to a considerable degree" 33 . Y aunque en la mayoría de los casos, dadas las condiciones en las que se lleva a cabo la aplicación del principio de proporcionalidad, no es posible llegar a una

\footnotetext{
29 BRINK (1988) p. 138.

30 BRINK (1988) p. 145.

31 Alexy (2006) p. 19.

32 Alexy (2006) pp. 22 y 24.

33 Alexy (2005) p. 580.
} 
única respuesta correcta ${ }^{34}$, Alexy no descarta que ello sea posible en condiciones ideales ${ }^{35}$. Como señala Kumm, la teoría de la argumentación constitucional de Alexy es claramente cognoscitiva: "there are sound or correct arguments. And there are right and wrong answers"

Ahora bien, ¿cómo aportan estas ideas a solucionar el problema de la legitimidad del control judicial de la ley? La respuesta que brinda Alexy es un poco atípica, pero en el fondo no muy distinta a la de Brink. Considera, en términos generales, que el control judicial es legítimo porque, al igual que los procedimientos legislativos, constituye también una forma de representación democrática ${ }^{37}$. Sin embargo, a diferencia de la representación que ejercen las cámaras del órgano legislativo, los tribunales no ejercen una representación volitiva (volitional), basada en la elección mediante voto de los representantes, sino más bien una "representación argumentativa": los jueces representan a los ciudadanos cuando fundamentan sus decisiones mediante razones. En ese sentido, el gobierno democrático no solo es electoral, sino también deliberativo ${ }^{38}$. Alexy considera $-y$ este es el punto esencial- que esta forma de representación, no basada en elecciones, es posible siempre que se cumplan dos condiciones: primero, que los fallos de los jueces estén sustentados en una aplicación correcta y racional de la constitución y, luego, que los argumentos aducidos por los jueces en los fallos puedan ser aceptados a largo plazo por personas razonables ${ }^{39}$, es decir, por personas que "are able and willing to accept sound or correct arguments for the reason that they are sound or correct" ${ }^{\prime 4}$. De esa manera, la legitimidad del control de constitucionalidad depende claramente de una concepción cognoscitivista de la interpretación: si existen respuestas correctas, los jueces están en la capacidad de hallarlas y los ciudadanos de aceptarlas, entonces el control de constitucionalidad es legítimo ${ }^{41}$.

\section{DWORKIN Y LA LECTURA MORAL DE LA CONSTITUCIÓN}

En Los Derechos en Serio, DwORKIN reconoce que los jueces pueden enfrentar casos difíciles (hard cases), es decir, casos para los cuales ninguna regla del sistema prevé una solución específica, sea porque el lenguaje de la regla está indeterminado o porque las soluciones disponibles son inadecuadas ${ }^{42}$. Pero el hecho de que las normas establecidas por el legis-

\footnotetext{
34 AleXY (2006) p. 22: "en la realidad no existe ningún procedimiento que permita, con una seguridad intersubjetivamente necesaria, llegar en cada caso a una única respuesta correcta”.

35 Alexy (2006) p. 23.

36 Kumm (2012) p. 206.

37 AlEXY (2005) p. 578: "the only way to reconcile constitutional review with democracy is to conceive of it, too, as representation of the people”. Una crítica a esta tesis es desarrollada por Kumm (2012).

38 AlEXY (2005) p. 579.

39 En sus palabras, "Constitutional review can be successful only if the arguments presented by the constitutional court are sound and only if a sufficient number of members of the community are able and willing to exercise their rational capacities" AleXY (2005) p. 580. Kumm, quien no comparte esta posición, lo expresa en los siguientes términos: "If the court's arguments are sound and if, in exercising their rational capacities, over time people are persuaded by the correct arguments presented by the court, the case for judicial review succeeds". Kumm (2012) p. 580.

40 Alexy (2005) p. 580

41 ALEXY (2005) p. 581: "If there exist sound and correct arguments as well as rational persons, reason and correctness are better institutionalized with constitutional review than without it".

42 DwORKIn (1989) p. 146.
} 
lador, o consagradas en el precedente, no establezcan una solución para el caso, no implica según Dworkin que la decisión del juez sea necesariamente discrecional ${ }^{43}$. Los sistemas jurídicos están conformados no solo por reglas, sino también por principios que suplen la carencia de reglas y que pueden ser desarrollados por los jueces como "questions of normative jurisprudence", es decir, como cuestiones de filosofía moral ${ }^{44}$. Y aunque Dworkin reconoce que los principios suelen ser, debido a su carácter moral, altamente controvertidos, y los jueces reales son a menudo incapaces de identificarlos y suelan equivocarse (con excepción del juez Hércules, una figura ideal con conocimientos jurídicos y de teoría política excepcionales, con la capacidad de encontrar siempre la respuesta correcta), existe -casi- siempre una respuesta mejor o más correcta que todas las demás ${ }^{45}$.

Dworkin ha bosquejado en algunos escritos las implicaciones metateóricas de esta postura. De manera muy resumida, considera que (i) todos los enunciados interpretativos en general, y las proposiciones jurídicas en particular, son siempre enunciados valorativos sujetos a controversias ${ }^{46}$; (ii) es posible diferenciar las buenas de las malas interpretaciones; y (iii) la interpretación, entendida como una práctica social, aspira siempre a encontrar las mejores interpretaciones, es decir, a encontrar la verdad ${ }^{47}$. Vista en estos términos, la teoría de la interpretación propuesta por Dworkin es claramente cognoscitivista. En su opinión, incluso, el escepticismo interpretativo (interno) es una de las varias posturas normativas que pueden asumirse respecto a la interpretación de una disposición jurídica específica: "una opinión escéptica solo es una interpretación diferente. Se apoya en supuestos sobre el valor en la misma medida que cualquiera de las interpretaciones positivas que cuestiona" ${ }^{38}$.

Ahora bien, la interpretación constitucional refleja cada una de estas características y Dworkin da cuenta de ella a través la denominada lectura moral de la constitución. Dworkin reconoce que algunas de las cláusulas constitucionales "are drafted in exceedingly abstract moral language", por lo que deben ser entendidas "in the way their language most naturally suggest: they refer to moral abstract principles and incorporate these by reference, as limits on government's power" ${ }^{49}$. Pero, nuevamente, la índole moral de los principios constitucionales no le resta objetividad a la interpretación, dado que existirían al menos dos restricciones importantes. Por una parte, los jueces deben interpretar la constitución aten-

\footnotetext{
43 DwOrkin (1963) p. 637: "in very hard cases, as in very easy ones, "judicial discretion" misdescribes judicial obligation".

44 DwOrKIN (1963) p. 636

45 Ver Dworkin, (1989) p. 177. De acuerdo con Dworkin, "aun en casos difíciles, los jueces tienen la obligación de determinar cuál es la respuesta correcta prevista por el Derecho... El hecho de que no exista un procedimiento preciso para determinar la respuesta correcta no implica que esta no exista". Ver también DwORKIN (1989) p. 146. Según Atienza, "...la tesis de DwOrKin es que siempre -o casi siempre- existe única respuesta correcta para cada caso, porque siempre hay una teoría sustantiva de carácter moral y político... que es la mejor, la más profunda”. ATIENZA (2016) p. 59.

46 DWORKIN (1985) p. 162.

47 DwORKIN (2014) p. 132: "cuando proponemos una interpretación de algo enunciamos y se entiende que enunciamos lo que tomamos como la verdad de un asunto". Ver también Dworkin (1985) p. 162

48 Dworkin (2014) p. 132.

49 Dworkin (1996) p. 7.
} 
diendo a la historia, "to answer the question of what they [the framers] intended to say" otra parte, los jueces deben obedecer el requerimiento de la integridad, garantizando que su interpretación sea consistente "with the structural design of the Constitution as a whole, and also with the dominant lines of past constitutional interpretation by other judges" ${ }^{51}$. Cumpliendo estos dos requerimientos, los jueces estarían en condiciones de alcanzar la mejor respuesta para el caso.

Ahora bien, Dworkin es además un conocido defensor del control judicial de la ley. $\mathrm{Su}$ argumento gira en torno al concepto de "democracia constitucional", un modelo de gobierno mayoritario limitado conceptualmente por condiciones morales sustantivas. En su opinión, la protección de los derechos constitucionales está mejor en manos de los jueces que en manos de las mayorías democráticas ${ }^{52}$, y una de las razones para ello -entre otras- es que los jueces están en mejores condiciones que los legisladores para hallar las respuestas correctas en la constitución. Cuando los jueces fundamentan sus decisiones en argumentos de principio, la objeción democrática no tiene sentido, puesto que los jueces no están tomando decisiones sobre la base de sus convicciones o intereses personales, sino con base en las condiciones sustantivas de la democracia previstas en la constitución. Dworkin supone aquí, de cierta manera, que las condiciones de la democracia (sobre todo los derechos) previstas en las cláusulas constitucionales son accesibles al razonamiento judicial. También asume que es precisamente esa capacidad de los jueces para identificar en el texto constitucional las condiciones de la democracia lo que en principio permite atribuirle mayor legitimidad en el control de constitucionalidad ${ }^{53}$. Refiriéndose a un caso ficticio en el que la Corte Suprema anula una norma que sanciona la quema de banderas por transgredir principios democráticos, Dworkin sostiene que "If the court's decision is correct-if laws against flag-burning do in fact violate the democratic conditions set out in the constitution as these has been interpreted and formed by American history- the decision is not antidemocratic, but, on the contrary, improves democracy".

Es en parte por estas razones que al momento de evaluar la legitimidad de una institución Dworkin termina por adoptar una forma de sustantivismo, que sustenta la dignidad moral de las decisiones políticas no tanto en el procedimiento, sino ante todo en el resultado sustantivamente correcto que pueden arrojar. "The best institutional structure", señala Dworkin, "is the one best calculated to produce the best answer to the essentially moral question of what the democratic conditions actually are, and to secure stable compliance with those conditions". Por eso, en su opinión, cuando las instituciones mayoritarias no respetan las condiciones democráticas (y aquí supone, claramente, que esas condiciones son bien conocidas por jueces y ciudadanos), "or when their provision or respect is defective, there can be no objection, in the name of democracy, to other procedures that protect and respect them better" ${ }^{54}$, tal como ocurriría con el control judicial de la ley.

\footnotetext{
50 DWORKIN (1996) p. 10.

51 DWORKIN (1996) p. 10.

52 DWORKIN (1996) p. 17.

53 DwOrkin (1996) p. 32.

54 Dworkin (1996) p. 34.
} 


\section{ESCEPTICISMO Y CONTROL DE CONSTITUCIONALIDAD}

Las teorías de Brink, Alexy y Dworkin son solo tres ejemplos notables del frecuente respaldo que goza la segunda premisa del argumento de Hamilton en la teoría del derecho. Algo similar sucede también, sin embargo, con quienes defienden teorías escépticas de la interpretación. La aparente inexistencia de respuestas correctas en la interpretación de las cláusulas constitucionales ha sido utilizada con frecuencia para objetar la legitimidad del control judicial de las leyes. El argumento es bastante simple: si la interpretación no arroja certeza acerca del contenido y el alcance de los derechos, entonces las decisiones de los jueces tendrán un contenido esencialmente moral y político, pero no jurídico ${ }^{55}$. Y puesto que, por una variedad de razones ${ }^{56}$, entre todos los procedimientos para la toma de decisiones que involucran asuntos políticos y morales son precisamente los democráticos los que poseen mayor legitimidad, es claro que existirían razones para negar a una élite de jueces la competencia para establecer -al menos con el carácter de última instancia- el contenido de los derechos constitucionales ${ }^{57}$. Estas consideraciones suponen, dado su carácter escéptico, una doctrina emotivista (o antirrealista) de la moral que explique por qué una decisión jurisdiccional con un contenido esencialmente ético no puede ser verdadero ni falso. La idea general del escepticismo es, como indica Waldron, la siguiente: "si la decisión de un juez contiene un elemento moral esencial, y si el realismo moral es falso, entonces el sentido de esta limitación desaparece. Los resultados estarán determinados por las preferencias subjetivas del juez, y en la medida en que sus actitudes se encuentren bajo su control, podrá tomar la decisión que quiera. Lo cual nos lleva naturalmente a la cuestión de por qué debería ser el juez, y no cualquier otra persona, el que haga lo que quiera en ese ámbito" 58 .

Muchos autores que objetan la supremacía judicial y defienden una mayor apertura democrática en cuestiones constitucionales asumen precisamente alguna forma de escepticismo interpretativo. Mark Tushnet, por ejemplo, ha sostenido insistentemente que el pueblo debe tener la última palabra acerca de la interpretación de las cláusulas constitucionales y que la interpretación que los jueces hacen de la constitución debe estar subordinada a la

\footnotetext{
55 Prieto (2009) p. 16: "en las críticas al constitucionalismo la tesis de la discrecionalidad suele presentar una fuerte tendencia a desembocar en un realismo extremo, de manera que la Constitución viene a ser poco más que un trozo de papel sin significado, una norma vaga, un dúctil instrumento que sirve para casi todo, repleto de preceptos vagos, porosos, contradictorios y que permiten los más arengados juegos de malabarismo interpretativo". Den Otter: "A legal decision that is reached exclusively for non-legal reasons is also illegitimate inasmuch as dissenters have not been given sufficient reasons to comply with it". DEN OTTER (2003) p. 376.

56 Citando a Waldron y a Laporta, Bayón ilustra algunas de las razones de mayor peso: "la idea de que la participación de todos y en pie de igualdad en la toma de decisiones públicas está revestida de una especial calidad moral, al margen del tipo de resultados a los que conduzca, parece gozar de una considerable fuerza intuitiva, porque hay "una cierta dignidad en la participación y un elemento de insulto [...] en la exclusión" y porque, al atribuir igual valor a la decisión de cada uno, el procedimiento de decisión por mayoría simple "traduce a su ámbito de aplicación [el] principio ético de la igualdad moral de todos los seres humanos como personas". ver BAYÓN (2010) p. 33.

57 Como señalan Redish y ARnould (2012) p. 1486: "If we ultimately conclude that neither the Constitution's text nor its history restrains unrepresentative, unaccountable judges in checking the actions of the political branches of either federal or state governments, little will be left of our system of popular sovereignty".

58 WALDRON (2005) p. 221.
} 
voluntad popular ${ }^{59}$, señalando a su vez que la interpretación constitucional no es de ninguna manera una labor cognoscitiva que restrinja los juicios políticos y morales de los jueces, sino una actividad esencialmente retórica: "No theory of interpretation, when fully elaborated, actually offered the prospect of contraining judges. Talented judges, if forced to adhere to the rules of any specified theory of interpretation, could produce whichever result they wanted any$w^{\prime \prime}{ }^{\prime 60}$. Así, por tanto, no existiendo certeza acerca del contenido de la constitución, ni un procedimiento interpretativo que permita alcanzar respuestas correctas, todo diseño institucional basado en el principio de supremacía judicial sería ilegítimo.

\section{LEGITIMIDAD, DESACUERDOS Y CONTROL DE CONSTITUCIONALIDAD}

El argumento de Hamilton trata de mostrarnos que la anulación o inaplicación judicial de las leyes creadas por el legislador democrático pueden ser legítimas cuando están fundamentadas en una interpretación correcta de la constitución. En esta sección pretendo mostrar que este argumento es incorrecto, pero antes haré un par de puntualizaciones sobre el concepto de legitimidad política y sobre las condiciones bajo las cuales una autoridad puede adquirirla.

¿Qué es la legitimidad política? En la teoría política suelen distinguirse los conceptos normativos de los conceptos descriptivos o sociológicos de legitimidad ${ }^{61}$. Desde el punto de vista descriptivo, como señala Peter $^{62}$, decimos que una autoridad es legítima cuando los miembros de la comunidad creen que existen razones morales para obedecer sus mandatos, es decir, cuando de hecho las personas aceptan que el ejercicio del poder político por parte de la autoridad se encuentra moralmente justificado. Desde el punto de vista normativo, por su parte, una autoridad tiene legitimidad política cuando efectivamente existen razones morales para obedecer sus mandatos, es decir, cuando la obediencia a la autoridad se encuentra moralmente justificada (no simplemente cuando las personas creen que existen esas razones). En la discusión que sigue asumiré un concepto normativo y no descriptivo de autoridad política, es decir, un concepto que alude a las razones morales que justifican que algunas personas o instituciones tomen decisiones por y para otros, y no a las creencias morales que tienen las personas acerca de la justificación de los procesos de toma de decisiones ${ }^{63}$.

Ahora bien, ¿en qué pueden consistir esas razones morales que justifican el ejercicio del poder político? En la teoría política contemporánea existen al menos tres grandes respuestas a este interrogante ${ }^{64}$. La primera señala que un proceso de decisión política es legítimo cuando las decisiones adoptadas son sustancialmente correctas, sin importar las carac-

\footnotetext{
59 Ver Tushnet (1992) p. 756 y (1999).

60 Tushnet (1992) p. 750.

61 Sobre el concepto de autoridad legítima, ver Peter (2017), Buchanan (2002) p. 689 y Martí (2006) p. 133.

62 Peter (2017).

63 Un concepto normativo que, como señalan Enoch y Fallon, es el que el mismo WaLdron inevitablemente debe asumir en los argumentos que mencionaré más adelante. Ver ENOCH (2006) p. 32 y FALLON (2008) p. 1717.

${ }^{64}$ Ver Linares (2008) p. 30 y MarTí (2006, p. 139).
} 
terísticas del procedimiento a través del cual fueron adoptadas ${ }^{65}$. Desde este punto de vista, que llamaremos "sustantivismo", lo que hace legítima a una autoridad es que el contenido de las decisiones satisfaga ciertas condiciones de justicia, como cuando reconoce o protege los derechos fundamentales. La segunda respuesta, por su parte, señala que el ejercicio del poder político de toma de decisiones es legítimo siempre que el procedimiento mismo materialice valores como el de la igual consideración y respeto para todos los ciudadanos. Desde este punto de vista, que llamaremos "procedimentalismo", lo que hace legítima a una autoridad no es el valor moral plasmado en el contenido de la decisión, sino exclusivamente el valor moral del proceso a través del cual esta fue adoptada. Hoy es ampliamente aceptado, sin embargo, que tanto el sustantivismo como el procedimentalismo -así explicadas- son teorías inadecuadas de la legitimidad política. Como señalan Martí y Linares ${ }^{66}$, el sustantivismo es inadecuado al menos por tres razones, que no explicaré en profundidad aquí: confunde la teoría de la legitimidad con la teoría de la justicia -"solo son legítimas las decisiones justas"-, cuando desde el punto de vista normativo y conceptual son diferentes ${ }^{67}$; está en condiciones de justificar los regímenes autoritarios, puesto que un dictador puede tomar decisiones sustancialmente justas; y excluye la posibilidad de que existan desacuerdos, dado que los principios de justicia suelen ser universales y entonces el parámetro de legitimidad es siempre y en todo lugar el mismo. Por su parte, el procedimentalismo es inadecuado porque no advierte que el valor de cualquier procedimiento depende en últimas de un conjunto de condiciones sustantivas que, entre otras cosas, nos permiten distinguir los buenos de los malos procedimientos ${ }^{68}$; no admite que esas condiciones sustantivas, cuando son transgredidas por el mismo procedimiento, pueden restar legitimidad a las decisiones (convirtiéndose así, por tanto, en condiciones sustantivas de legitimidad); y, en su versión más radical ${ }^{69}$, asume la idea contraintuitiva de que el procedimiento es en sí mismo constitutivo de las verdades morales.

Es por estas razones, entre otras, que parece más adecuada la tercera respuesta: una teoría mixta de la legitimidad. "Una decisión política para ser legítima”, sostiene Martî70, "debe haber sido tomada siguiendo un procedimiento determinado reconocido como legítimo, y ser además respetuosa en algún sentido con ciertos valores sustantivos de justicia”. Por supuesto, una teoría mixta de la legitimidad no está exenta de problemas: si el valor del procedimiento y el valor sustantivo de la decisión entran en conflicto, ‘a cuál se le debe dar prioridad? Buena parte de la teoría política de las últimas décadas se ha dedicado a dar

\footnotetext{
65 Martí (2006) p. 141.

66 Martí (2006) p. 142 y Linares (2008) p. 31.

${ }^{67}$ Como señala Waldron, "una teoría política... necesita ser más que una teoría de la justicia o una teoría del bien común. También tiene que tratar la cuestión normativa sobre la legitimidad de los procesos de toma de decisiones que son utilizados para realizar decisiones políticas frente a los desacuerdos". WALDRON (2015) p. 174. Ver también: BAYÓN (2002) p. 87.

68 Como han puesto de manifiesto Christiano y Bayón, el carácter sustantivo y controvertido de esas condiciones hace que el procedimientalismo desarrollado por Waldron sea inconsistente. Ver Christiano (2000), p. 520 y BAYÓN (2002) p. 120.

${ }^{69}$ Valentini (2012) p. 6.

70 Martí (2006) p. 148.
} 
respuesta a este y a otros interrogantes. Sin embargo, aquí no me ocuparé de estas discusiones. Lo único que me interesa ahora es analizar cómo el argumento de Hamilton, particularmente su segunda premisa (cognoscitivismo), así como el argumento propuesto por el escepticismo interpretativo, tratan de proporcionar las razones que justifican el control judicial de la ley sobre una base sustantivista o procedimentalista.

Por lo que hemos visto hasta ahora, es posible construir al menos dos argumentos sugerentes con base en el cognoscitivismo presupuesto por Hamilton. En primer lugar, podríamos decir que no existen objeciones importantes a la legitimidad del control judicial de la ley, porque las decisiones de los jueces se limitan a reproducir la voluntad del pueblo. Desde este punto de vista, si suponemos que el pueblo se ha pronunciado democráticamente $-\mathrm{y}$ además que, por ser muy reciente la constitución, no tiene suficiente fuerza la objeción intertemporal (el gobierno de los muertos sobre los vivos), entonces ni siquiera surge la objeción democrática al control judicial, dado que las decisiones judiciales, siendo correctas, reflejarían las preferencias morales o políticas del pueblo y no las de los jueces. En segundo lugar, podríamos decir, alternativamente, que no existen objeciones importantes porque las decisiones judiciales, siendo correctas, reflejan las condiciones indispensables para materializar el ideal de la democracia o el ideal de la justicia (aun cuando no correspondan, plenamente, a la voluntad que el pueblo consignó en la constitución).

El primero de estos argumentos es procedimentalista: supone que la decisión de anular o inaplicar una ley es legítima porque emana, en última instancia, de un procedimiento democrático. La corrección sustantiva de las decisiones no sería, por tanto, un requisito indispensable para respaldar la legitimidad del control judicial de la ley. Más aún, preguntarse por la legitimidad política de la decisión del juez podría carecer de sentido, dado que su función no consistiría estrictamente en la toma de decisiones políticas, sino en la reproducción de las que previamente ha tomado el pueblo. El segundo argumento, por su parte, es sustantivista: sostiene que las decisiones de los jueces, aun cuando no correspondan exactamente con las intenciones o los propósitos que se planteó originalmente el pueblo, resguardan y promueven las condiciones necesarias para que exista democracia y justicia en la comunidad (y aquí ya nos alejamos, sutilmente, de la primera premisa del argumento de Hamilton).

Por su parte, el escepticismo también dispondría de un argumento bastante sugerente. Si el resultado de la interpretación constitucional no refleja otra cosa que las preferencias morales y políticas de los jueces, y no es posible, por razones conceptuales, que la constitución limite de forma efectiva el sentido de esas decisiones, entonces el control judicial es ilegítimo. Entre todos los procedimientos disponibles no sería precisamente el judicial el más legítimo. Como señala Prieto Sanchís: "en la tensión entre los derechos y la democracia, quienes se inclinan decididamente en favor de esta última han de rechazar como es lógico cualquier forma de control judicial, máxime si se abraza con entusiasmo la tesis de la discreción judicial" 71 . Esta crítica al control de constitucionalidad, sustentada en una posición escéptica de la interpretación, supone claramente una teoría procedimentalista de la legitimidad.

\footnotetext{
1 Prieto Sanchís (2009) p. 158.
} 
Me parece que estos argumentos constituyen, en términos generales, el marco sobre el cual se ha discutido muchas veces la relación entre la interpretación constitucional y la legitimidad del control judicial de la ley. En Estados Unidos y otros países, por ejemplo, buena parte de las discusiones entre originalistas y no originalistas descansa sobre la idea de que existen respuestas correctas en la interpretación constitucional y que la labor de los jueces y filósofos es descubrir los instrumentos y procedimientos adecuados para encontrar$\operatorname{las}^{72}$. De igual manera, como hemos visto, quienes objetan el control judicial suelen considerar como una razón contundente el hecho de que la constitución es tan indeterminada que no ofrece (casi) nunca respuestas correctas para los casos concretos. Me parece que hay, sin embargo, buenas razones para creer que la existencia o inexistencia de respuestas correctas no tiene suficiente relevancia para fundamentar u objetar la legitimidad del control judicial de la ley, y que, por tanto, los argumentos que acabamos de analizar son profundamente defectuosos.

El gran problema con estos argumentos es que desnaturalizan la discusión sobre la legitimidad política. Suponen que la corrección sustancial de las decisiones es suficiente para justificar moralmente a la institución que las adopta, o que la inexistencia de respuestas correctas basta para objetarla. De esta manera olvidan, como señala Waldron, que la mayoría de las veces el problema de la legitimidad no consiste en tomar la decisión correcta, o en hallar la forma de llegar a ella, sino en cómo es posible superar los múltiples e irreductibles desacuerdos que existen entre los miembros de la comunidad acerca de cuales son las respuestas correctas y cómo podemos llegar a ellas ${ }^{73}$. Según Waldron ${ }^{74}$, los problemas de legitimidad surgen solo cuando concurren las "circunstancias de la política", es decir, cuando los miembros de la comunidad están divididos por desacuerdos profundos y de buena fe acerca del bien y de la justicia, y necesitan tomar decisiones que permitan la acción común. $\mathrm{Y}$ aunque en ocasiones la existencia de desacuerdos sobre el contenido y el alcance de los derechos parece ser consecuencia de la verdad del escepticismo, e igualmente esperaríamos que no existieran desacuerdos profundos si el cognoscitivismo fuese verdadero, "it's the disagreements themselves, and not their metaethical or metaphysical characterization, that give rise to problems of legitimacy concerning judicial review"75. Veamos por qué.

En primer lugar, la existencia de respuestas correctas no tiene suficiente relevancia para justificar el control judicial de la ley (y de cualquier otro procedimiento de toma de decisiones políticas). Como señala Waldron, "there is no logical connection between objective moral truth and justification of the practice of judicial review of popular legislation" ${ }^{\text {" }}$. (Waldron habla aquí de verdades morales, en el sentido acuñado por el realismo metaético, y no directamente de interpretaciones verdaderas de la constitución, pero es claro que esta última idea se encuentra implícita en la primera: dado que para Waldron el lenguaje de los derechos previsto en la constitución tiene un carácter netamente moral, las interpretaciones

\footnotetext{
72 Ver Gargarella (2012) p. 79.

73 Ver Waldron (1998) y Waldron (2005), Goldini (2012) p. 930, Bayón (2002) p. 87 y Valentini (2012) p. 6.

74 WALDRON (2005) p. 123.

75 Waldron (1998) p. 79. Ver también MarTí (2006) p. 136.

76 WALDRON (1998) p. 76.
} 
"verdaderas" de las cláusulas constitucionales corresponderían a verdades morales). No es cierto, por tanto, que una decisión sea legítima siempre que sea correcta, es decir, siempre que sea moralmente verdadera o pueda deducirse de la constitución. Y no lo es porque, aun siendo verdadero el cognoscitivismo, entre los mismos ciudadanos existen profundas controversias que versan precisamente sobre cuál es la respuesta correcta y cuáles son los criterios o procedimientos que permiten diferenciar las respuestas correctas de las incorrectas. Ante un desacuerdo, lo único que nos dice el cognoscitivismo es que algunos ciudadanos están equivocados y otros no, pero no proporciona una epistemología fiable, verificable intersubjetivamente, que permita diferenciar entre unos y otros. Es por eso que tras la supuesta relación lógica que existe entre el cognoscitivismo y la justificación moral del control judicial se esconde en últimas una teoría de la legitimidad que no tiene reparo en que los jueces impongan sus propias creencias acerca del sentido verdadero de los derechos, aun cuando los demás ciudadanos, actuando de buena fe, tengan opiniones muy diferentes y no comprendan siquiera qué características hacen que la decisión de los jueces sean objetivamente correctas.

Estas mismas conclusiones se seguirían si en vez de limitarnos a aceptar que existen respuestas correctas en la interpretación de los derechos sostenemos al mismo tiempo que el proceso judicial tiene valor epistémico, es decir, que son precisamente los jueces quienes están mejor capacitados para hallar las respuestas (objetivamente) correctas. Dworkin ha señalado varias veces que las mejores (o verdaderas) interpretaciones de los derechos son más accesibles a los jueces que a las asambleas democráticas por varias razones: los jueces tratan de tomar decisiones sobre la base de principios y no de cálculos instrumentales, no están sujetos a compromisos políticos como sí lo están los legisladores, y estimulan una mayor discusión pública sobre problemas de moralidad política ${ }^{77}$. Sin embargo, esta tesis sucumbe también a los argumentos señalados en el párrafo anterior. Por una parte, entre los ciudadanos existen profundos desacuerdos acerca de cuál es la mejor interpretación de los derechos, por lo que en principio no podríamos identificar objetivamente ${ }^{78}$ cuál es el procedimiento que conduce más probablemente a ella ${ }^{79}$. Por otra parte, las mismas características que en opinión de Dworkin constituyen una virtud del procedimiento judicial han sido objeto de fuertes críticas: por ejemplo, los jueces suelen prestar demasiada atención a los problemas de interpretación del texto y no directamente, como sería lo ideal, a las cuestiones de moralidad política asociadas a los derechos ${ }^{80}$. Finalmente, y aún más importante, para que la tesis sobre el valor epistémico del procedimiento judicial permita justificar el control judicial

\footnotetext{
77 DwORKIN (1985) p. 25: "legislators are subject to pressures that judges are not, and this must count as a reason for supposing that ... judges are more likely to reach sound conclusions about rights". Ver también DwORKIN (1996) p. 30.

78 Esto no implica, por supuesto, que los ciudadanos no puedan evaluar el valor instrumental del procedimiento con base en sus creencias personales sobre el valor de la decisión. Ver BAYÓN (2002) p. 119 y FALLON (2008), p. 1703. Me parece, sin embargo, que en ese caso no es adecuado atribuirle al procedimiento un "valor epistémico", dado que este presupone la existencia de criterios objetivos para evaluar la verdad o corrección de la decisión.

79 Esta tesis es desarrollada en detalle por Valentini, para quien el procedimiento democrático no puede tener valor epistémico cuando entre los ciudadanos existen desacuerdos razonables y robustos (thick reasonable disagreements). VAlentini (2012) p. 15. Para un análisis crítico de esta idea, ver GerLsbeCK (2016).

${ }^{80}$ Ver Waldron (2015) p. 87 y Waldron (2009) p. 22.
} 
de las leyes, no bastarían las teorías metaéticas y semánticas que respalden la existencia de respuestas correctas, pues serían necesarias también las investigaciones empíricas que muestren, por una parte, que son los jueces quienes las hallan con mayor facilidad y, por otra, que una vez las hallan deciden sin embargo no desatenderlas ${ }^{81}$.

En segundo lugar, la inexistencia de respuestas correctas tampoco tiene suficiente relevancia para objetar el control judicial de la ley. Ante un desacuerdo, el escepticismo nos dice que ninguno de los ciudadanos posee una respuesta objetivamente correcta, pero nada más. Y aunque a simple vista esto parezca hablar en favor de los procedimientos democráticos y en contra del control judicial, en el fondo no lo hace ${ }^{82}$. La razón por la que atribuimos una especial dignidad a la democracia no es que carezcamos, en un plano metafísico o semántico, de respuestas correctas en asuntos morales y políticos, sino que existen múltiples e irreconciliables posturas acerca de cuáles son esas respuestas correctas. El escepticismo es perfectamente compatible con la idea de que las decisiones que involucran derechos sean adoptadas, en ciertos contextos, por los jueces: si no existen hechos morales o entidades semánticas objetivas, pero entre los ciudadanos existen acuerdos profundos sobre el contenido y el alcance de los derechos, entonces no existirían objeciones importantes al control de constitucionalidad ${ }^{83}$. No es por tanto la inexistencia de respuestas correctas sino los desacuerdos entre los ciudadanos lo que genera, nuevamente, la necesidad de diseñar procedimientos legítimos de decisión.

La conexión entre el escepticismo y la ilegitimidad del control judicial de la ley es a veces sugerente justamente porque tendemos a creer, de forma equivocada, que los desacuerdos son consecuencia de la inexistencia de respuestas correctas. Sin embargo, sabemos que es conceptualmente posible que existan amplios consensos sociales respecto del contenido de los derechos y que no existan pese a ello respuestas correctas en un sentido metaético o semántico. En casos como este no tendría mucho sentido objetar el control de judicial de la ley sosteniendo que la decisión del juez será, ante la ausencia de respuestas correctas, necesariamente discrecional. En realidad, la existencia de un consenso social sobre el contenido "correcto" de la decisión hace que las preocupaciones por el valor intrínseco (e incluso instrumental) de la democracia disminuyan y se puedan considerar legítimos otros procedimientos de toma de decisiones. Esa es la intuición que probablemente compartieron Kelsen y Bayón ${ }^{84}$ cuando sugieren que una constitución compuesta por reglas y no por principios puede hacer frente a la objeción democrática al control judicial de la ley: el control sería le-

81 WaLdRON (1998) p. 83: "Facts do not constrain in the sense of constraint that interests us in politics. That there is a right answer "out there" certainly means that a judge is not making a fool of himself when he goes out ponderously in search of it. But its mere existence does not drive the judge to pursue it, let al.one determine that he will reach it".

82 WALDRON (2015) p. 171: "Uno puede reconocer la existencia del desacuerdo sobre cuestiones de derechos y justicia... sin plantear la afirmación meta-ética de que no existe la verdad en el asunto acerca del problema respecto del que disputan los participantes. El reconocimiento del desacuerdo es perfectamente compatible con la existencia de una verdad sobre el asunto de los derechos y los principios del constitucionalismo".

83 VAlentini (2012) p. 10. Incluso, la autora considera que el valor de la democracia pierde fuerza cuando existen desacuerdos irrazonables (unreasonable disagreements).

84 Ver Kelsen (1928, ver edición española 2011) p. 287 y BAYÓn (2001) p. 84. Una posición similar es desarrollada por Verdugo, para quien la existencia en Chile de una constitución "con mayor densidad normativa" en comparación con la estadounidense, y "que entrega más respuestas normativas en cuestiones donde existen 
gítimo porque el contenido y el alcance de las reglas constitucionales sería, en comparación con los principios, menos susceptible de profundas controversias sociales, y no tanto porque se pueda encontrar en las reglas (y no en los principios) la respuesta correcta.

La cuestión acerca de si existen o no respuestas correctas no tendría, por tanto, consecuencias prácticas: sea asumiendo una posición escéptica o una posición cognoscitivista, los desacuerdos acerca del contenido y el alcance de los derechos fundamentales subsistirían. Lo que en realidad importa es tomar en serio las circunstancias de la política, que exigen que las decisiones sean tomadas a través de procedimientos legítimos, y esa legitimidad no depende de la existencia o inexistencia de respuestas correctas, sino del valor de los procedimientos. Los problemas relacionados con el diseño de las instituciones políticas, entre ellas los órganos encargados de interpretar los derechos constitucionales, no pueden reducirse a meras disputas acerca de la existencia objetiva de hechos morales o entidades semánticas. Como señala Waldron, "el realista moral no está mejor ubicado que el emotivista a la hora de legitimar el control judicial de constitucionalidad. Y lo contrario también es válido: el emotivista no está peor situado que el realista a este respecto. Esta disputa se debe ganar sobre la base de los méritos morales o políticos, sobre la base de argumentos morales de equidad, de justicia y democracia" ${ }^{\prime 5}$.

En la mayoría de sociedades contemporáneas, una de las circunstancias de la política más recurrentes son los desacuerdos sobre el contenido y el alcance de los derechos constitucionales. La opinión de los ciudadanos se encuentra a menudo dividida entre bandos opuestos frente a grandes controversias que involucran cláusulas constitucionales: ¿la pornografía está protegida por el derecho a la libre expresión? ¿El derecho a la autonomía personal implica el derecho de las mujeres a abortar? ¿Hace uso de su derecho al libre desarrollo de la personalidad quien consume marihuana? ¿El derecho a la igualdad implica que las parejas homosexuales puedan contraer matrimonio? El aspecto más destacable de estas controversias, sin embargo, es que parecen estar sustentadas siempre en la buena fe de quienes debaten. Los defensores y detractores del aborto, por ejemplo, suelen citar principios de moralidad política para respaldar sus posiciones y conocer a cabalidad las razones que sus contrincantes pretenden hacer valer en favor de las suyas. No se trata, por tanto, de un desacuerdo ilegítimo, originado en la hipocresía o autointerés de quienes discuten, sino en convicciones honestas acerca del sentido profundo de los derechos ${ }^{86}$.

Los desacuerdos son comunes incluso entre los mismos jueces que ejercen el control de constitucionalidad. Al interior de los tribunales colegiados las decisiones se toman casi siempre por mayoría y, cuando involucran cuestiones moralmente sensibles, es bastante inusual la unanimidad. En estos casos, los jueces que disienten de la mayoría suelen formular -cuando es posible- salvamentos de voto (dissenting opinions) en los que exponen de manera ponderada y honesta las razones que en su opinión hacen incorrecta la decisión adoptada

desacuerdos morales", hace que se debiliten las objeciones de Waldron al control judicial de la ley. VerdUGO (2013) p. 210.

85 WaLdRON (2005) p. 222.

86 WaLdRON (2015) p. 171: "es innegable que las disposiciones de la carta de derechos hacen referencia al asunto, pero lo que implican y si ellas prohíben (o deben limitar la aplicación de) las disposiciones legislativas que están en tela de juicio, sigue siendo cuestión de disputa entre personas razonables". 
por la mayoría. Entre esas razones no solo están las que atribuyen en un primer nivel contenidos diferentes a los derechos, sino también las que en un segundo nivel censuran los procedimientos o técnicas de interpretación utilizadas en la sentencia. Es por este mismo sistema de votación mayoritario que a veces se produce, además, uno de los principales síntomas de desacuerdo al interior de los tribunales: cuando cambian las mayorías, es común que las nuevas decisiones anulen los precedentes adoptados por el mismo tribunal en el pasado.

\section{CONCLUSIONES}

La idea de que existen respuestas correctas en la interpretación de derechos ha sido utilizada por algunos autores para justificar el control judicial de la ley, mientras que otros autores, partidarios del escepticismo interpretativo o el emotivismo ético, consideran que es justamente la ausencia de respuestas correctas el fundamento esencial de la objeción democrática. En este trabajo traté de mostrar, sin embargo, que estos argumentos están mal construidos. Dado que los ciudadanos suelen disentir de forma honesta y razonada sobre el la interpretación correcta de los derechos constitucionales, el cognoscitivismo en realidad no ofrece por sí solo ninguna respuesta a los interrogantes básicos de la legitimidad (¿cómo y quién debe decidir cuál es la respuesta correcta?), y el escepticismo no ofrece tampoco una razón importante para objetar que los jueces, en ausencia de desacuerdos, puedan ser quienes tomen finalmente la decisión.

Una teoría de la legitimidad del control judicial que tome en serio la circunstancia política de los desacuerdos debe estar basada al menos en parte en el mérito moral y político de los procedimientos. Con esto no quiero sugerir, por supuesto, que para evaluar la legitimidad de la justicia constitucional solo sean relevantes los principios morales que inspiran el procedimiento y podamos prescindir del contenido de las decisiones. Solo quiero destacar que cuando utilizamos las teorías de la interpretación, sobre todo el cognoscitivismo, para fundamentar u objetar determinados diseños institucionales, en realidad perdemos de vista el hecho de que el valor de las decisiones políticas depende, al menos en parte, de las personas y los procesos que hayan intervenido en su adopción.

Tampoco quiero sugerir, finalmente, que la negación de cualquier nexo lógico entre los problemas semánticos o metaéticos y los problemas de legitimidad despeja de alguna manera el camino para negar toda legitimidad al control judicial de la ley. Admitir que ante los desacuerdos ninguna teoría sobre el significado de las cláusulas constitucionales tiene suficiente relevancia para evaluar la legitimidad del control judicial solo implica que la verdadera discusión debe versar sobre argumentos que respondan adecuadamente a los desafíos de la legitimidad. Y entre todos esos argumentos tendrán mayor peso, probablemente, los que honren de mejor manera el principio de igualdad de trato y respeto para todos.

\section{BIBLIOGRAFÍA CITADA}

Alexy, Robert (2005): “Balancing, Constitutional Review, and Representation”, International Journal of Constitutional Law, vol 3, No 5: pp. 572-581. 
AleXY, Robert (2006): "Ponderación, control de constitucionalidad y representación" en IbÁÑeZ, Perfecto y Alexy, Robert (edit.), Jueces y Ponderación Argumentativa (México, UNAM).

Alexy, Robert (2006a): Derecho y razón práctica (México, Fontamara).

Alexy, Robert (2007): Teoría de los derechos fundamentales (Madrid, Centro de Estudios Políticos y Constitucionales).

AtienZa, Manuel (2016): Interpretación Constitucional (Bogotá, Universidad Libre).

BAYÓN, Juan Carlos (2001): "Derechos, democracia y constitución”, Discusiones, vol 1: pp. 65-94.

BAYÓN, Juan Carlos (2002): "Democracia y Derechos: problemas de fundamentación del constitucionalismo", en Betegón, Jerónimo; Laporta, Francisco; De PÁramo, Juan Ramón y Prieto SAnchís, Luis (edit.), Constitución y derechos fundamentales (Madrid, Centro de Estudios Políticos y Constitucionales).

BeItz, Charles (1989): Political Equality. An Essay in Democratic Theory (Princeton, Princeton University Press).

Christiano, Thomas (2000): "Waldron on Law and Disagreement". Law and Philosophy, Vol. 19: pp. 513-543.

Dahl, Robert (1989): Democracy and its Critics (New Haven, Yale University Press).

Den Otter, R. (2004). “The place of Moral judgement in Constitutional interpretation”, Indiana Law review, Vol 37, No. 2: pp. 375-416.

DwORKIN, Ronald (1985): A matter of Principle (Cambridge, Harvard University Press).

DwOrkIN, Ronald (1989): Los derechos en serio (Barcelona, Ariel).

Dworkin, Ronald (1996): Freedom's Law (Oxford, Oxford University Press).

Dworkin, Ronald (2014): Justicia para erizos (México, Fondo de Cultura Económica).

Enoch, David (2006): “Taking Disagreement Seriously: On Jeremy Waldron's Law and Disagreement”, Israel Law Review, Vol. 39, No 3: pp. 22-35.

Elster, John (1988): Ulysses and the Syrens (Cambridge, Cambridge University Press).

Fallon, Richard (2008): "The Core of An Uneasy Case for Judicial Review", Harvard Law Review, Vol 121: pp. 1693-1723.

Ferrajoli, Luigi (2003): "Sobre la definición de "democracia". Una discusión con Michelanagelo Bovero”, Isonomía, vol 19: pp. 227-240.

Ferrajoli, Luigi (2013): Principia Iuris. Teoría del derecho y de la democracia, Tomo II: Teoría de la democracia (Madrid, Trotta).

Gargarella, Roberto (1997): "La dificultad de defender el control judicial de las leyes", Isonomía, vol 6: pp. 55-70.

Gargarella, Roberto (2012): La justicia frente al gobierno. Sobre el carácter contramayoritario del poder judicial (Quito, Corte Constitucional de Ecuador).

GerlsBeCK, F (2016): "What is democratic reliability? Epistemic theories of democracy and the problem of reasonable disagreement", Critical Review of International Social and Political Philosophy, 2016: pp. 1-23.

Goldini, Marco (2012): "Two internal critiques of political constitutionalism", $I \bullet C O N$, vol 10, No 4: pp. 926-949. 
Hamilton, Alexander (2005): "The Federalist 78: A View of the Constitution of the Judicial Department, in Relation to the Tenure of Good Behaviour", en Hamilton, Alexander; Madison, James y JaY, John (eds.) The Federalist (Indianápolis, Hackett Publishing Company) pp. 411-418.

Kelsen, Hans (2011): "La garantía jurisdiccional de la constitución (la justicia constitucional)", Anuario Iberoamericano de Justicia Constitucional, No 15: pp. 249-300.

Kumm, Matthias (2012): "Alexy's Theory of Constitutional Rights and the Problem of Judicial Review”, en KLATT, Matthias (Edit), Institutionalized reason. The jurisprudence of Robert Alexy (Oxford, Oxford University Press) pp. 201-217.

LinARES, Sebastián (2008): La (i)legitimidad del control de constitucionalidad (Madrid, Marcial Pons).

Marmor, Andrei (2015): “Son legitimas las constituciones?”, en FABra Zamora, Jorge Luis y García Jaramillo, Leornardo (edit.), Filosofía del derecho constitucional. Cuestiones fundamentales (México, UNAM) pp. 33-67.

Martí, José Luis (2006): La república deliberativa (Madrid, Marcial Pons).

Redish, Martin H. y Arnould, Matthew B. (2012): "Judicial review, constitutional interpretation, and the democratic dilemma: proposing a "controlled activism" alternative", Florida Law Review, vol 64, No 6: pp. 1486-1537.

Peter, Fabienne (2017): "Political Legitimacy [Stanford Encyclopedia of Philosophy]". Disponible en: https://plato.stanford.edu/entries/legitimacy/. Fecha de consulta: 10 de noviembre de 2019.

PRIETO SANChÍs, Luis (2009): Justicia constitucional y derechos fundamentales (Madrid, Trotta).

Ramírez Ludeña, Lorena (2015): Diferencias y deferencia. Sobre el impacto de las nuevas teorías de la referencia en el derecho (Madrid, Marcial Pons).

Tushnet, Mark (1992): "Constitutional Interpretation, Character, and Experience", Boston University Law Review, vol 72: pp. 747-763.

Tushnet, Mark (1999): Taking the constitution away from the courts (Princeton, Princeton University Press).

VAlEntini, Laura (2012): "Justice, disagreement and democracy", British Journal of Political Science, 2012: pp. 1-23.

Verdugo, Sergio (2013): "La discusión democrática sobre la revisión judicial de las leyes de diseño institucional y modelos constitucionales", Revista Chilena de Derecho, vol. 40 No 1: pp. 181-223.

Waldron, Jeremy (1998): "Moral Truth and Judicial Review", The American Journal of Jurisprudence, vol 43, No 1: pp. 75-97.

Waldron, Jeremy (2005): Derecho y desacuerdos (Madrid, Marcial Pons).

WaLdron, Jeremy (2009): “Judges as moral reasoners", $I \bullet C O N$, vol 7, No 1: pp. 2-24.

WALDRON, Jeremy (2015): "El centro de la argumentación contra el control judicial de la legislación”, en Fabra Zamora, Jorge Luis y García Jaramillo, Leonardo (edit.), Filosofía del derecho constitucional. Cuestiones fundamentales (México, UNAM) pp. 147-2014. 